\title{
Value of SiPM PET in myocardial perfusion imaging using Rubidium-82
}

\author{
S. S. Koenders, PhD (D, ${ }^{\mathrm{a}, \mathrm{d}}$ J. A. van Dalen, PhD, ${ }^{\mathrm{b}}$ P. L. Jager, $\mathrm{MD}, \mathrm{PhD}{ }^{\mathrm{a}}$ \\ S. Knollema, MD, MSc, PhD, ${ }^{\mathrm{a}}$ J. R. Timmer, MD, PhD, ${ }^{\mathrm{C}} \mathrm{M}$. Mouden, $\mathrm{MD}, \mathrm{PhD},{ }^{\mathrm{c}} \mathrm{C}$. \\ H. Slump, PhD, ${ }^{\text {d }}$ and J. D. van Dijk, MSc, PhD (1) ${ }^{a}$ \\ a Department of Nuclear Medicine, Isala Hospital, Zwolle, The Netherlands \\ b Department of Medical Physics, Isala Hospital, Zwolle, The Netherlands \\ c Department of Cardiology, Isala Hospital, Zwolle, The Netherlands \\ d Technical Medical Centre, University of Twente, Enschede, The Netherlands
}

Received Jan 27, 2020; accepted Apr 3, 2020

doi: $10.1007 / \mathrm{s} 12350-020-02141-0$

Background. PET scanners using silicon photomultipliers with digital readout (SiPM PET) have an improved temporal and spatial resolution compared to PET scanners using conventional photomultiplier tubes (PMT PET). However, the effect on image quality and visibility of perfusion defects in myocardial perfusion imaging (MPI) is unknown. Our aim was to determine the value of a SiPM PET scanner in MPI.

Methods. We prospectively included 30 patients who underwent rest and regadenosoninduced stress Rubidium-82 (Rb-82) MPI on the D690 PMT PET (GE Healthcare) and within three weeks on/ the Vereos SiPM PET (Philips Healthcare). Two expert readers scored the image quality and assessed the existence of possible defects. In addition, interpreter's confidence, myocardial blood flow (MBF), and myocardial flow reserve (MFR) values were compared.

Results. Image quality improved $(P=\mathbf{0 . 0 3})$ using the Vereos as compared to the D690. Image quality of the Vereos and the D690 was graded fair in $20 \%$ and $10 \%$, good in $60 \%$ and $50 \%$, and excellent in $20 \%$ and $40 \%$, respectively. Defect interpretation and interpreter's confidence did not differ between the $D 690$ and the Vereos $(P>0.50)$. There were no significant differences in rest MBF $(P \geq 0.29)$, stress MBF $(P \geq 0.11)$, and MFR $(P \geq 0.51)$.

Conclusion. SiPM PET provides an improved image quality in comparison with PMT PET. Defect interpretation, interpreter's confidence, and absolute blood flow measurements were comparable between both systems. SiPM PET is therefore a reliable technique for MPI using Rb-82.

Trial registration. ToetsingOnline NL63853.075.17. Registered 13 November, 2017. (J Nucl Cardiol 2022;29:204-12.)

Key Words: SiPM PET $\cdot$ Rb-82 $\cdot$ myocardial blood flow $\cdot$ PET $\cdot$ myocardial perfusion imaging

\footnotetext{
Electronic supplementary material The online version of this article (https://doi.org/10.1007/s12350-020-02141-0) contains supplementary material, which is available to authorized users.

The authors of this article have provided a PowerPointfile, available for download at SpringerLink, which summarizes thecontents of the paper and is free for re-use at meetings andpresentations. Search for the article DOI on SpringerLink.com.
}

Reprint requests: S. S. Koenders, PhD, Department of Nuclear Medicine, Isala Hospital, Zwolle, The Netherlands; skoenders93@gmail.com 1071-3581/\$34.00

Copyright (C) 2020 The Author(s) 


\begin{tabular}{|ll|}
\hline Abbreviations & \\
CT & Computed tomography \\
LAD & Left anterior descending \\
LCX & Left circumflex \\
LV & Left ventricle \\
MBF & Myocardial blood flow \\
MFR & Myocardial flow reserve \\
MPI & Myocardial perfusion imaging \\
PET & Positron emission tomography \\
Rb-82 & Rubidium-82 \\
TAC & Time activity curve \\
\hline
\end{tabular}

See related editorial, pp. 213-215

\section{INTRODUCTION}

Myocardial perfusion imaging (MPI) using positron emission tomography (PET) is increasing in popularity over single photon emission computed tomography (SPECT) in the last years due to the increased availability of strontium-82/rubidium-82 (Rb-82) generators, higher spatial resolution, and higher sensitivity and specificity. ${ }^{1}$ In addition, PET enables quantification of myocardial blood flow (MBF), which provides valuable additional prognostic information about the extent and functional importance of possible stenosis over visual assessment. $^{2-4}$

Recently, new PET systems using silicon photomultipliers with digital readout (SiPM PET) have become available for clinical use. ${ }^{5-8}$ In terms of system performance, the SiPM PET design results in an improved spatial and timing resolution and a relatively high count-rate capability as compared to PET scanners using conventional photomultiplier tubes (PMT PET). ${ }^{5-7,9,10}$ First oncology-PET studies showed that SiPM PET provides an improved image quality over PMT PET. ${ }^{10-13}$ However, studies demonstrating the value of SiPM PET for MPI are still lacking. Hence, our aim was to determine the value of SiPM PET in comparison with PMT PET in MPI using Rb-82.

\section{MATERIALS AND METHODS}

\section{Study Design}

We performed a prospective single-center study and included 30 consecutive patients referred for MPI using PMT PET (Discovery 690, GE Healthcare; D690) with $\mathrm{Rb}-82$ for the evaluation of coronary artery disease. Within three weeks after the first PET scan, patients underwent a second MPI PET scan on a SiPM PET scanner (Vereos, Philips Healthcare). The local institutional ethics committee approved the study protocol and informed consent was obtained from all individual participants included in the study.

\section{Patient Preparation and Data Acquisition}

Patients were asked to refrain from caffeine containing beverages for at least $24 \mathrm{~h}$ before both scans. All patients underwent a rest scan followed by a regadenoson-induced stress scan on both scanners. First, a lowdose computed tomography (CT)-scan was performed for attenuation correction purposes. The CT scan on the D690 was performed using $0.8 \mathrm{~s}$ rotation time, pitch of 0.97 , collimation of $32 \times 0.625 \mathrm{~mm}$, tube voltage of $120 \mathrm{kV}$, and tube current of $10 \mathrm{~mA}$. On the Vereos, the CT scan was acquired using $1.5 \mathrm{~s}$ rotation time, pitch of 0.83 , collimation of $64 \times 0.625 \mathrm{~mm}$, tube voltage of $120 \mathrm{kV}$, and tube current of $22 \mathrm{~mA}$. The PET acquisition protocol was similar for the D690 and Vereos. A fixed activity of $740 \mathrm{MBq} \mathrm{Rb}-82$ was intravenously administered with a flow rate of $50 \mathrm{~mL} / \mathrm{min}$ using a strontium82/Rb-82 generator (CardioGen-82, Bracco Diagnostics Inc.) immediately followed by a seven-minute PET acquisition. Ten minutes after the first activity bolus, stress was pharmacologically induced by administering $400 \mu \mathrm{g}(5 \mathrm{~mL})$ regadenoson over 10 seconds. After a 5 $\mathrm{mL}$ saline flush $(\mathrm{NaCl} 0.9 \%)$, the second activity bolus of $740 \mathrm{MBq}$ was administered followed by a sevenminute stress PET acquisition. To obtain patient's effective radiation dose for both PET examinations we used the conversion factors of $0.00126 \mathrm{mSv} / \mathrm{MBq}$ for rest and $0.00128 \mathrm{mSv} / \mathrm{MBq}$ for stress ${ }^{14,15}$ resulting in a total dose of $1.9 \mathrm{mSv}$. To calculate the effective dose for the attenuation CT, we used a conversion factor of 0.014 $\mathrm{mSv} /(\mathrm{mGy} \cdot \mathrm{cm})^{16}$ resulting in $0.2 \mathrm{mSv}$ for the D690 based on an average dose length product (DLP) of 11.8 $\mathrm{mGy} \cdot \mathrm{cm}$ and $0.8 \mathrm{mSv}$ for the Vereos based on an average DLP of $60.5 \mathrm{mGy} \cdot \mathrm{cm}$.

\section{Image Reconstruction}

CT data associated with the D690 were reconstructed using an iterative reconstruction method (70\% adaptive statistical iterative reconstruction algorithm, ASIR) and a slice thickness of $5 \mathrm{~mm}$. CT data associated with the Vereos were reconstructed using an iterative reconstruction method (iDose level 4) and a slice thickness of $3 \mathrm{~mm}$.

We applied attenuation correction to all acquired PET data after semi-automatic registration of the CT and PET using the PET data acquired between 2:30 and 7:00 minutes. ${ }^{17}$ We reconstructed the images of the D690 with a 3D-ordered subset expectation maximization (OSEM) technique using 2 iterations and 24 subsets and 
a Gaussian post-smoothing filter of $12 \mathrm{~mm}$, as recommended by the manufacturer. The voxel size of the D690 was $3.3 \times 3.3 \times 3.3 \mathrm{~mm}^{3}$. Images of the Vereos were reconstructed with $3 \mathrm{D}$ OSEM using 3 iterations and 15 subsets and a Gaussian post-smoothing filter of $6 \mathrm{~mm}$. The voxel size of the Vereos was $4.0 \times 4.0 \times 4.0 \mathrm{~mm}^{3}$. These Vereos settings were determined prior to our study (see appendix), based on measurements using an anthropomorphic torso phantom with a cardiac insert (model ECT/TOR/P, Data Spectrum Corp.). Intensity profiles through the cardiac insert were collected for several reconstruction settings to compare the full width at half maximum value to that of the D690. This way we obtained reconstruction settings resulting in an equivalent image resolution. For both the D690 and the Vereos data, corrections were performed for decay, scatter and random coincidences, and dead time effects. We used data acquired from 2:30 to 7:00 minutes for both rest and stress scans to obtain static images. Dynamic data sets were reconstructed using 26 time frames $(12 \times 5$ seconds, $6 \times 10$ seconds, $4 \times 20$ seconds and $4 \times 40$ seconds). All reconstructed images were postprocessed using Corridor4DM software (v2016).

\section{Visual Assessment}

Each set of static rest and stress PET images, showing the relative perfusion, was analyzed by two expert readers in consensus. They scored the image quality, visibility of perfusion defects, and interpreter's confidence. Image quality of the static images was assessed using a four-point grading scale: 1) poor, 2) fair, 3) good, and 4) excellent. Readers assessed the image quality based on myocardial count density and uniformity in well-perfused areas, signal to background noise, and shape of the left ventricle (LV). Static images were visually characterized as normal or abnormal. Abnormal scans were characterized as ischemic and/or irreversible. The interpreter's confidence was scored as either definite or equivocal. Readers were unaware of the patient's history or other clinical findings. Images were presented in random order and readers were blinded for the PET system.

\section{MBF Quantification}

Activity concentrations were measured in the 26 reconstructed time frames to calculate the time activity curves (TACs) for the LV, for the three vascular territories: left anterior descending (LAD), left circumflex (LCX) and right coronary (RCA) artery, and for the whole myocardium (global). The one-tissue compartment model of Lortie et al. based on a ROI methodology was used to calculate the MBF from the TACs. ${ }^{18}$ Rest
MBF was calculated without rate-pressure product correction. Furthermore, myocardial flow reserve (MFR) was calculated as the ratio between the stress and rest MBF. We categorized the global MFR values into three categories: high risk on cardiac failure with MFR $<1.5$, intermediate risk with MFR between 1.5 and 2.0, and low risk with MFR $>2.0 .^{2,19}$

Rest or stress MBF and MFR values were excluded from the comparison evaluation in case of unreliable TACs. Unreliable TACs were defined as TACs without a clear LV peak during the first-pass phase or a lack of steady state for the three vascular territories during the tissue phase, as previously described. ${ }^{20}$ Test-retest precision was calculated as the standard deviation (SD) of the relative MBF and MFR differences, as previously defined by Kitkungvan et al. ${ }^{21}$ A test-retest precision $\leq 21 \%$ was considered acceptable. ${ }^{21}$

\section{Statistical Analysis}

Patient-specific parameters and characteristics were determined as mean $\pm \mathrm{SD}$, or as percentages using SPSS (IBM SPSS Statistics for Windows, Version 24.0). Image quality, MBF and MFR measurements were compared using the Wilcoxon signed rank test. In addition, the visibility of perfusion defects and interpreter's confidence were compared using the McNemar test. The level of statistical significance was set to 0.05 for all statistical analyses.

\section{RESULTS}

\section{Baseline Characteristics}

The baseline characteristics of the included patients are summarized in Table 1.

\section{Visual Assessment}

Image quality of the static images improved $(P=0.03)$ using the Vereos as compared to the D690. Image quality of the D690 and the Vereos was graded fair in 20\% (6/30) and 10\% (3/30), good in 60\% (18/30) and 50\% (15/30), and excellent in 20\% (6/30) and $40 \%$ $(12 / 30)$, respectively, as illustrated in Figure 1. None of the images using either the D690 or the Vereos were scored as poor. An example of the image quality for patients with high and low BMI is shown in Figure 2.

Defect interpretation did not differ in $93 \%$ (28/30) of the patient scans between the D690 and the Vereos $(P=0.50)$. In the $7 \%(2 / 30)$ of patient scans where defect interpretation differed, the scans were scored as normal on the D690, whereas they were interpreted to show ischemia on the Vereos (Figures 3 and 4). Patient 
Table 1. Baseline characteristics of all included patients $(\mathrm{N}=30)$ who underwent clinically indicated $\mathrm{Rb}-82$ PET MPI

\begin{tabular}{ll}
\hline Characteristic & All patients \\
\hline Age (years) & $64 \pm 9$ \\
Male $(\%)$ & 80 \\
Weight $(\mathrm{kg})$ & $87 \pm 15$ \\
Height $(\mathrm{cm})$ & $176 \pm 9$ \\
BMI $\left(\mathrm{kg} / \mathrm{m}^{2}\right)$ & $28.0 \pm 4.4$ \\
Current smoker (\%) & 13 \\
Hypertension (\%) & 50 \\
Diabetes (\%) & 20 \\
Dyslipidemia (\%) & 40 \\
Family history (\%) & 53
\end{tabular}

Data are presented as mean \pm SD or as percentage

scans were scored as normal in $80 \%(24 / 30)$ and $73 \%$ $(22 / 30)$ for the D690 and the Vereos, respectively. Furthermore, $10 \%(3 / 30)$ and $17 \%(5 / 30)$ was interpreted as showing ischemia and for both PET scanners $13 \%$ (4/30) was scored as showing an irreversible defect. There was no difference in interpreter's confidence as all scans were scored as definite.

\section{MBF Quantification}

Of the 30 included patients, both rest and stress MBF values of one patient were excluded due to unreliable rest and stress TACs. Furthermore, rest MBF values of another patient and stress MBF values of four other patients were excluded due to unreliable TACs. The main reason for an unreliable TAC was no clear or absent LV peak, which would be most likely caused by a pinched vein. ${ }^{22}$ The remaining PET scans provided a paired comparison of 28 rest MBF values, 25 stress MBF values, and 24 MFR values.

There were no significant differences in any of the vascular territories nor in the whole myocardium regarding the rest $\operatorname{MBF}(P \geq 0.29)$, stress $\operatorname{MBF}(P \geq$ $0.11)$ or MFR $(P \geq 0.51)$, as shown in Table 2 and Figure 5. When categorizing the global MFR values into high, intermediate, and low risk on cardiac failure, 25\% $(6 / 24)$ of the patients were reclassified when using the Vereos. More specifically, one patient was reclassified from intermediate risk to high risk, three from intermediate risk to low risk, and two patients from low risk to intermediate risk. None of the patients were reclassified from low risk to high risk or vice versa. Moreover, testretest precision of global rest $\mathrm{MBF}$, stress $\mathrm{MBF}$, and MFR was $18 \%, 16 \%$, and $21 \%$, respectively, and was

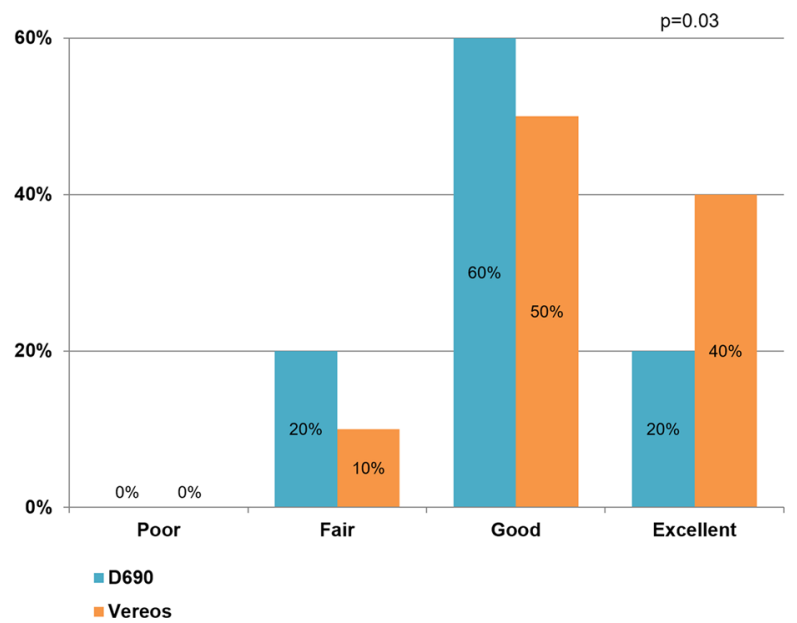

Figure 1. Barplot showing the percentage of images scored as poor, fair, good, and excellent for the D690 and Vereos PET system. Image quality improved for the Vereos $(P=0.03)$.

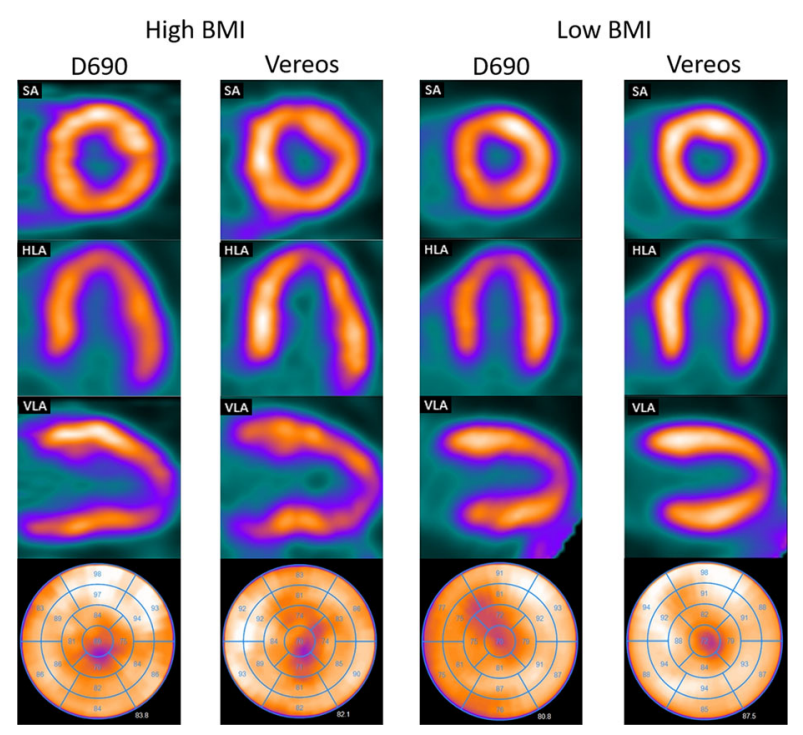

Figure 2. Example of the rest study from two patients scanned on the D690 and Vereos PET system. The images on the left are from a patient with a high BMI $\left(36.5 \mathrm{~kg} / \mathrm{m}^{2}\right)$ and on the right of a patient with a low BMI $\left(19.2 \mathrm{~kg} / \mathrm{m}^{2}\right)$. From top to bottom: SA; short axis, HLA; horizontal long axis, VLA; vertical long axis, bull's eye.

considered to be within the previously reported testretest precision of 21\%, as shown in Figure 6.

\section{DISCUSSION}

In this study, we showed that the Vereos SiPM PET scanner provided an improved image quality for MPI using Rb-82 as compared to the D690 PET scanner using conventional photomultiplier tubes. There were no 

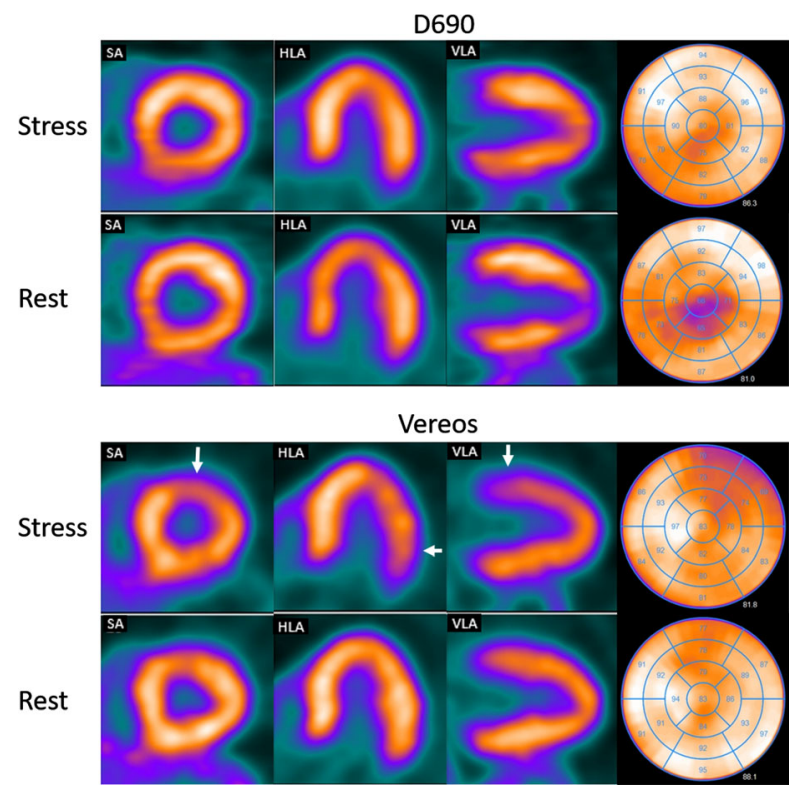

Figure 3. Example of a rest and stress study from the same patient scanned on the D690 (top) and Vereos (bottom). The images of the D690 show no defect while the images of the Vereos show a small reversible defect in the basal anterolateral wall (white arrows). From left to right: SA; short axis, HLA; horizontal long axis, VLA; vertical long axis, bull's eye.
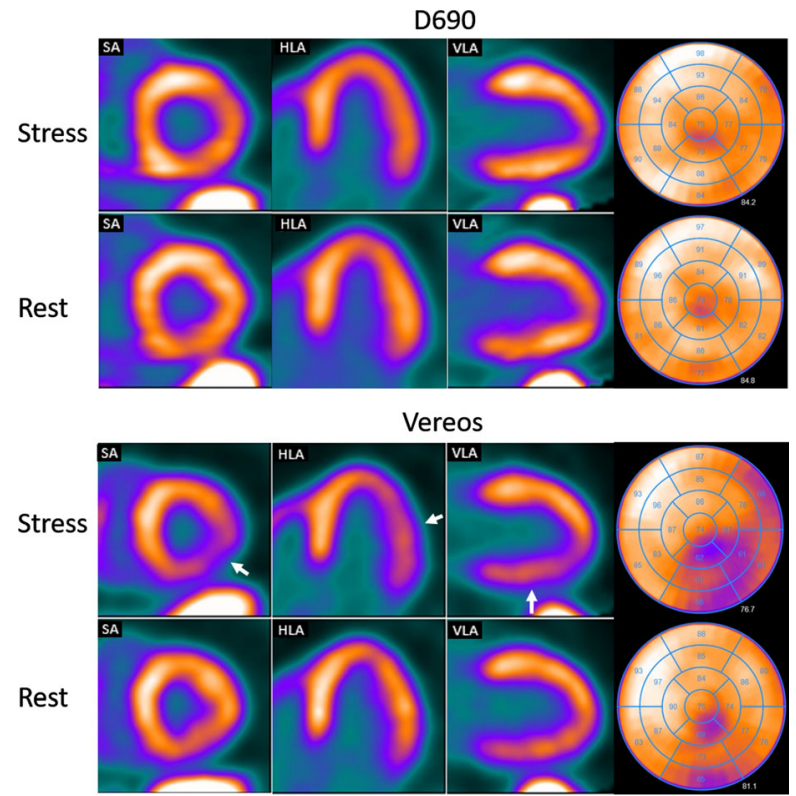

Figure 4. Example of a rest and stress study from the same patient scanned on the D690 (top) and Vereos (bottom). The images of the D690 show no defect while the images of the Vereos show a moderate reversible defect in the inferolateral wall (white arrows). From left to right: SA; short axis, HLA; horizontal long axis, VLA; vertical long axis, bull's eye.

significant differences in defect interpretation or in quantitative MBF and MFR measurements.
According to previously performed phantom and patients studies, ${ }^{6,10-13}$ SiPM PET showed an improved image quality and lesion detection for oncology patients as compared to PMT PET. It seems that these results can be generalized to cardiac imaging as shown in our study. In addition, scan interpretation might change as well when shifting from PMT PET to SiPM PET. In our population, images from two out of 30 patients showed ischemia on the Vereos PET scan, whereas these images were interpreted as normal on the D690 PET scan. Of these patients, one had no follow-up imaging and no events within the first year after the Vereos PET scan (Figure 3). Global MFR values of this patient were 2.15 using the D690 (low risk) and 1.87 using the Vereos (intermediate risk). In the other patient (Figure 4), a subtotal stenosis was seen in the circumflex area during coronary angiography one month after the second PET scan, corresponding to the ischemic area in the Vereos PET images. Global MFR values of this patient were 1.72 using the D690 (intermediate risk) and 1.40 using the Vereos (high risk). It is well known that perfusion defects can be introduced due to misregistration of attenuation CT and PET data. ${ }^{23-25}$ For each scan, we verified the co-registration between $\mathrm{CT}$ and PET data. In none of the scans, a misregistration was observed.

For MBF quantification, the Vereos showed reliable $\mathrm{MBF}$ and MFR measurements using Rb-82. This is in line with the results of the study by Van Dijk et al. who performed a cardiac-phantom study and concluded that the D690 and the Vereos scanner showed a comparable count-rate performance for Rb-82 activities up to approximately $1000 \mathrm{MBq} .{ }^{26}$ However, in our study, $25 \%(6 / 24)$ of the patients were reclassified according to the global MFR values from intermediate risk to low/ high risk or vice versa when shifting to the Vereos. The relative differences of these six patients were $38 \%, 15 \%$, $10 \%,-14 \%,-19 \%$ and $-21 \%$. Although $25 \%$ seems to be a large percentage, it is possible that a patient classified as having an intermediate risk may be classified as having a low/high risk when repeating the scan and reprocessing the data, solely due to the relatively large test-retest precision in MBF and MFR measurements of typically $21 \% .^{21}$ Therefore, reclassification in $25 \%$ of the patients is not solely due to the differences in scanner performance. Furthermore, test-retest precision was calculated as the SD of the relative MBF and MFR differences for all patients. Therefore, by definition, approximately $68 \%$ of all test-retest values should be within the test-retest precision of $21 \%$.

This study had several limitations that should be recognized. First, our study population was relatively small $(\mathrm{N}=30)$. Still, the interpreter's confidence was scored as definite in $100 \%$ of the scans so it is unlikely that including more patients will give a significant 
Table 2. Rest and stress $M B F(\mathrm{~mL} / \mathrm{min} / \mathrm{g})$ and $M F R$ values calculated for the $\mathrm{D} 690$ and the Vereos PET scans, for the three vascular territories (LAD, LCX, and RCA) and the whole myocardium (global). No significant differences were observed between the D690 and the Vereos PET $(P \geq 0.11)$

\begin{tabular}{lllll}
\hline Territory & PET scanner & Rest MBF $(\mathbf{N}=\mathbf{2 8})$ & Stress $\mathbf{M B F}(\mathbf{N}=\mathbf{2 5})$ & MFR $(\mathbf{N}=\mathbf{2 4})$ \\
\hline \multirow{2}{*}{ LAD } & D690 & $0.9[0.7-1.1]$ & $2.0[1.8-2.5]$ & $2.3[2.0-2.8]$ \\
& Vereos & $0.9[0.7-1.0]$ & $2.2[1.7-2.4]$ & $2.3[2.0-2.7]$ \\
\multirow{2}{*}{ LCX } & D690 & $0.9[0.7-1.1]$ & $2.2[1.9-2.5]$ & $2.3[2.0-2.9]$ \\
& Vereos & $0.9[0.7-1.1]$ & $2.1[1.6-2.3]$ & $2.3[1.8-2.7]$ \\
\multirow{3}{*}{ Global } & D690 & $0.9[0.7-1.1]$ & $2.2[2.0-2.5]$ & $2.3[2.0-3.0]$ \\
& Vereos & $0.9[0.7-1.2]$ & $2.3[2.0-2.9]$ & $2.4[1.9-3.3]$ \\
& D690 & $0.9[0.7-1.0]$ & $2.0[1.8-2.5]$ & $2.3[2.0-2.9]$ \\
& Vereos & $0.9[0.7-1.0]$ & $2.1[1.7-2.5]$ & $2.3[2.0-2.8]$ \\
\hline
\end{tabular}

Data are presented as median (interquartile range)

$L A D$, left anterior descending; $L C X$, left circumflex; $M B F$, myocardial blood flow; $M F R$, myocardial flow reserve; and $R C A$, right coronary artery
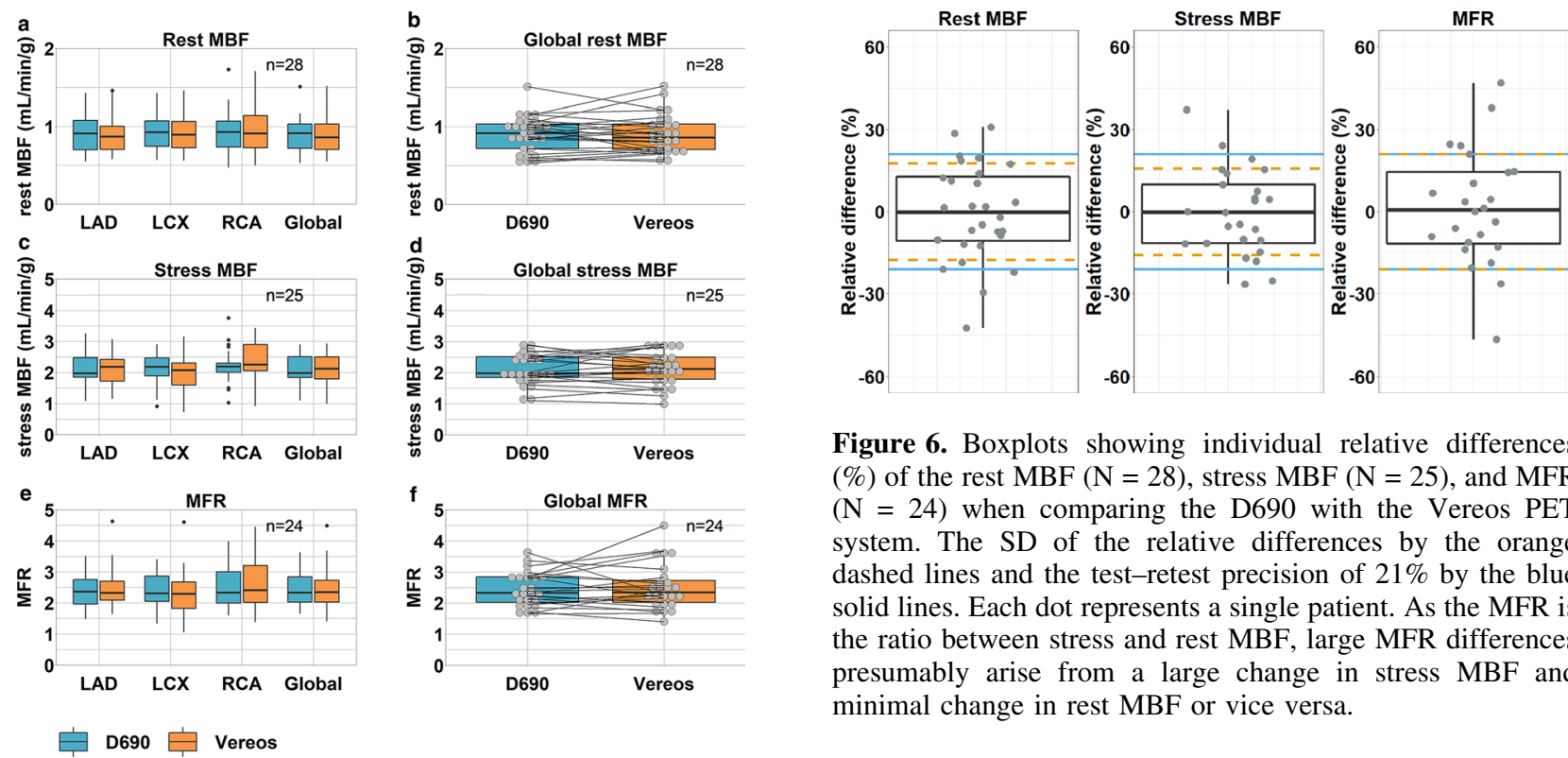

Figure 6. Boxplots showing individual relative differences $(\%)$ of the rest MBF $(\mathrm{N}=28)$, stress $\mathrm{MBF}(\mathrm{N}=25)$, and MFR $(\mathrm{N}=24)$ when comparing the D690 with the Vereos PET system. The SD of the relative differences by the orange dashed lines and the test-retest precision of $21 \%$ by the blue solid lines. Each dot represents a single patient. As the MFR is the ratio between stress and rest MBF, large MFR differences presumably arise from a large change in stress $\mathrm{MBF}$ and minimal change in rest $\mathrm{MBF}$ or vice versa.

the D690 PMT PET. Furthermore, we found relative MBF and MFR differences within the 21\% test-retest precision. It is not likely that this will change when more patients are included.

Secondly, some elements of the acquisition have to be addressed. As we used $\mathrm{Rb}-82$, the positron range (5.9 $\mathrm{mm})$ is rather large compared to for example ${ }^{13} \mathrm{~N}$ ammonia $(1.5 \mathrm{~mm})$, which results in a worse image resolution compared to using other PET MPI tracers. ${ }^{27}$ The higher spatial resolution of the SiPM PET as compared to PMT PET may therefore result in an even better image quality when using other PET tracers than $\mathrm{Rb}-82$. Moreover, the injected activity used in this study is lower than generally recommended $(740 \mathrm{MBq}$ vs. $1110 \mathrm{MBq}),{ }^{2}$ but sufficient for MBF quantification. ${ }^{26,28}$ 
In addition, we used a PMT PET scanner with a relatively high count-rate capability. Therefore, our results may not be generalizable to older PMT PET scanners as they might not be able to process the high count-rates adequately due to dead time effects. ${ }^{26,28}$ Inaccurate count-rate measurements can result in unreliable MBF and MFR measurements. ${ }^{29}$

Lastly, expert readers had no access to clinical information, i.e., gender, age, or calcium score when interpreting the images. Although there was no significant difference in defect interpretation, it still differed in two patients. Access to clinical information might have altered their decision-making and could have overcome this different interpretation.

\section{Clinical Implications}

The Vereos scanner was the first SiPM PET scanner available for clinical use ${ }^{6}$ after which two other SiPM PET scanners became available, namely, the Biograph Vision PET/CT (Siemens Healthineers) and the Discovery MI (GE healthcare). ${ }^{7,8}$ As the performance characteristics of SiPM PET are in general better that those of PMT PET, image quality is expected to improve for all three SiPM PET scanners. Moreover, flow measurements are expected to be similar or possibly more accurate as compared to using PMT PET, provided that PMT PET has a sufficient count-rate capability. ${ }^{7,8,26,28,29}$

Whereas the MFR was shown to be robust when using different advanced reconstruction settings or software packages, one should be cautious in the occurrence of possible systematic changes in MBF measurements. ${ }^{30,31}$ Furthermore, one has to be aware of a relatively large test-retest precision in MBF and MFR measurements of typically $21 \%$. In general, a MFR $<1.5$ is associated with an increased risk on cardiac failure while patients with a MFR $>2.0$ are associated with a reduced risk on cardiac failure. ${ }^{2,19}$ Hence, it is possible that a patient classified as having an intermediate risk may be classified as a low/high-risk patient when repeating the scan and reprocessing the data, solely due to the test-retest variation.

\section{New Knowledge Gained}

The use of the Vereos SiPM system in PET Rb-82 MPI results in an improved image quality and no significant differences for visual interpretation or interpreter's confidence in comparison with the conventional D690 PMT PET scanner. Furthermore, no significant differences were found in MBF and MFR quantification. We did find a change in visual defect interpretation in two patients. Defect interpretation may therefore differ and it could be possible that the Vereos SiPM PET system has a superior diagnostic performance over the conventional D690 PMT PET system. Additional studies with a larger patient population are required to confirm this.

\section{CONCLUSIONS}

PET using silicon photomultipliers with digital readout is a reliable technique for MPI using Rb-82 as it provides an improved image quality and similar interpreter's confidence, defect interpretation, and absolute blood flow measurements as compared to PET using conventional photomultiplier tubes.

\section{Disclosures}

This work was supported by a research grant of Philips Healthcare. The content of the article was solely the responsibility of the authors.

\section{Conflict of interest}

This work was supported by a research grant of Philips Healthcare. The content of the article was solely the responsibility of the authors.

\section{Ethical approval}

All procedures performed in studies involving human participants were in accordance with the ethical standards of the institutional and/or national research committee (METC Isala Zwolle, NL63853.075.17) and with the 1964 Helsinki declaration and its later amendments or comparable ethical standards.

\section{Informed consent}

Informed consent was obtained from all individual participants included in the study.

\section{Open Access}

This article is licensed under a Creative Commons Attribution 4.0 International License, which permits use, sharing, adaptation, distribution and reproduction in any medium or format, as long as you give appropriate credit to the original author(s) and the source, provide a link to the Creative Commons licence, and indicate if changes were made. The images or other third party material in this article are included in the article's Creative Commons licence, unless indicated otherwise in a credit line to the material. If material is not included in the article's Creative Commons licence and your intended use is not permitted by statutory regulation or exceeds the permitted use, you will need to obtain permission directly from the copyright holder. To view a copy of this licence, visit http://creativecommons.org/licenses/by/4.0/. 


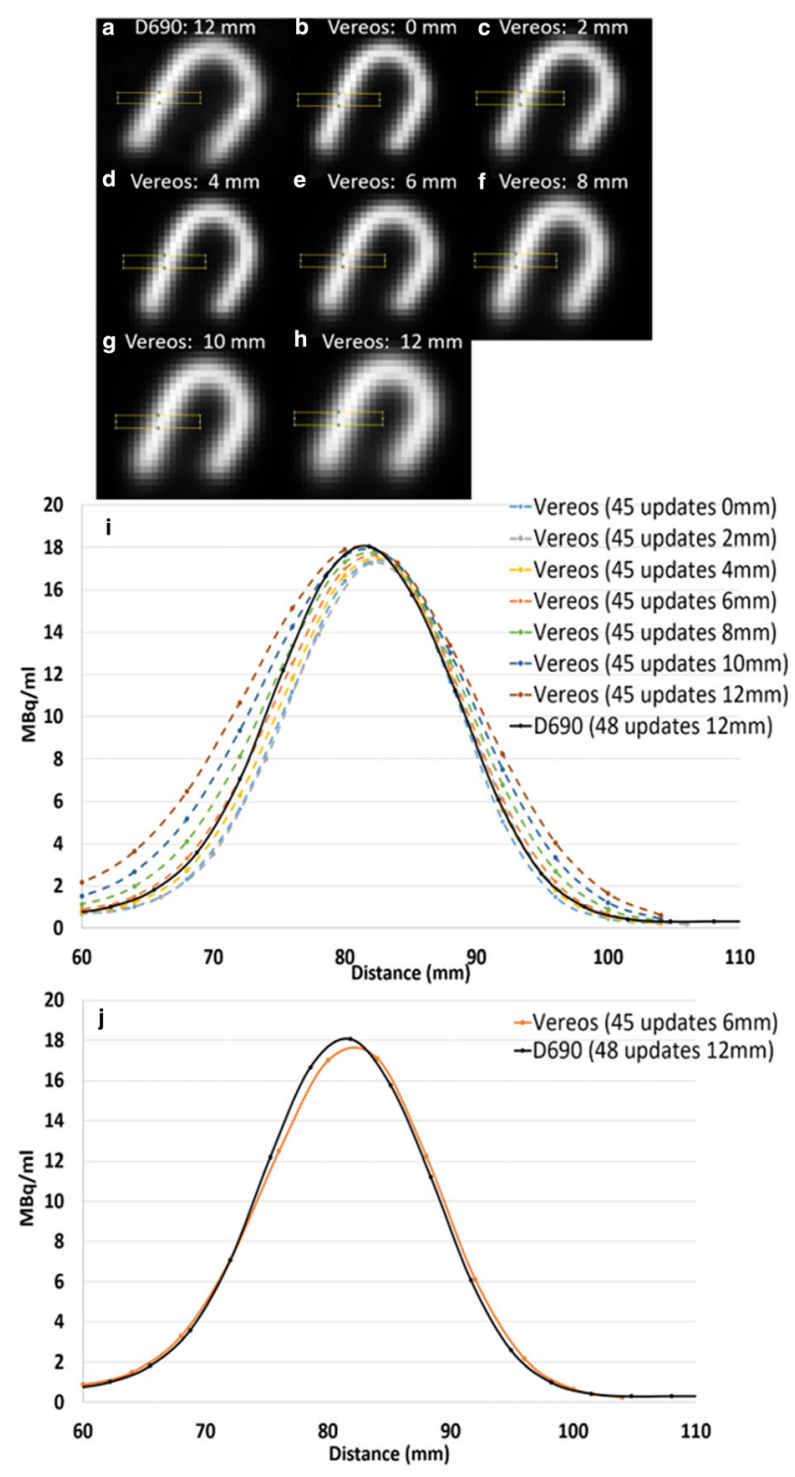

Figure 7. Reconstructed PET images of an $\mathrm{Rb}$-82-filled Cardiac Insert phantom using the D690 (A) with 2 iterations, 24 subsets and a Gaussian post-smoothing filter of $12 \mathrm{~mm}$ and using the Vereos (B-H) with 3 iterations, 15 subsets and Gaussian post-smoothing filter ranging from $0-12 \mathrm{~mm}$. The rectangles were used to plot intensity profiles. The width of the profile increases when increasing the filter for the Vereos reconstructions (I). The intensity profile reconstructed on the Vereos using 3 iterations, 15 subsets and a Gaussian postsmoothing filter of $6 \mathrm{~mm}$ is most similar to the profile using the image reconstruction of the D690 (J).

\section{APPENDIX}

\section{Aim}

We performed a phantom study to obtain reconstruction settings for the Vereos SiPM PET resulting in similar image resolution as compared to the Discovery 690 PMT PET.

\section{Method}

We used an anthropomorphic torso phantom with a cardiac insert (model ECT/TOR/P, Data Spectrum Corp.) which simulates myocardial uptake in the left ventricular chamber. An activity of $2590 \mathrm{MBq} \mathrm{Rb}-82$ was injected. The phantom was scanned on the D690 PET system (GE healthcare) and on the Vereos PET system (Philips Healthcare). PET data of the phantom were reconstructed with a 3D-ordered subset expectation maximization (OSEM) technique using 48 updates (2 iterations and 24 subsets) and a Gaussian post-smoothing filter of $12 \mathrm{~mm}$ on the D690, as recommended by the manufacturer. PET data of the phantom of the Vereos were reconstructed with OSEM using 45 updates (3 iterations and 15 subsets) and seven different Gaussian post-smoothing filters, with kernel-widths of $0,2,4,6,8$, 10 and $12 \mathrm{~mm}$. Intensity profiles through the images of the cardiac insert were collected for the seven reconstruction settings (Figure 7A-H) to compare the full width at half maximum value to that of the D690.

\section{Results}

With increasing filtering, the full width at half maximum value also increases (Figure 7I). Using a 6 $\mathrm{mm}$ filter in the image reconstruction of the Vereos data resulted in a similar full width at half maximum value as compared to the D690 (Figure 7J).

\section{Conclusion}

Reconstruction settings using 45 updates and a Gaussian post-smoothing filter of $6 \mathrm{~mm}$ were used to reconstruct the static and dynamic images of the patients who underwent rest and regadenoson-stress PET Rubidium- 82 on the Vereos.

\section{References}

1. Mc Ardle BA, Dowsley TF, deKemp RA, Wells GA, Beanlands RS. Does rubidium-82 PET have superior accuracy to SPECT perfusion imaging for the diagnosis of obstructive coronary disease?: A systematic review and meta-analysis. J Am Coll Cardiol 2012;60:1828-37.

2. Murthy V, Bateman T, Beanlands R, Berman D, Borges-Neto S, Chareonthaitawee $\mathrm{P}$, et al. Clinical quantification of myocardial blood flow using PET: Joint position paper of the SNMMI cardiovascular council and the ASNC. J Nucl Cardiol 2018;25:26997.

3. Ziadi M, deKemp R, Williams K, Guo A, Renaud J, Chow B, et al. Does quantification of myocardial flow reserve using rubidium- 82 positron emission tomography facilitate detection of multivessel coronary artery disease? J Nucl Cardiol 2012;19:670-80.

4. Sciagra R, Passeri A, Bucerius J, Verberne HJ, Slart, Riemer H. J. A., Lindner $\mathrm{O}$, et al. Clinical use of quantitative cardiac perfusion 
PET: Rationale, modalities and possible indications. position paper of the cardiovascular committee of the european association of nuclear medicine (EANM). Eur J Nucl Med Mol Imaging 2016;43:1530-45.

5. Slomka PJ, Pan T, Germano G. Recent advances and future progress in PET instrumentation. Semin Nucl Med 2016;46:5-19.

6. Miller M, Zhang J, Binzel K, Griesmer J, Laurence T, Narayanan $\mathrm{M}$, et al. Characterization of the vereos digital photon counting PET system. J Nucl Med 2015;56(supplement 3):434.

7. van Sluis JJ, de Jong J, Schaar J, Noordzij W, van Snick P, Dierckx R, et al. Performance characteristics of the digital biograph vision PET/CT system. J Nucl Med 2019;60:1031-6.

8. Hsu DFC, Ilan E, Peterson WT, Uribe J, Lubberink M, Levin CS. Studies of a next-generation silicon-Photomultiplier-Based timeof-flight PET/CT system. J Nucl Med 2017;58:1511-8.

9. Slomka PJ, Pan T, Berman DS, Germano G. Advances in SPECT and PET hardware. Prog Cardiovasc Dis 2015;57:566-78.

10. Van der Vos CS, Koopman D, Rijnsdorp S, Arends AJ, Boellaard $\mathrm{R}$, van Dalen JA, et al. Quantification, improvement, and harmonization of small lesion detection with state-of-the-art PET. Eur J Nucl Med Mol Imaging 2017;44:4-16.

11. López-Mora DA, Flotats A, Fuentes-Ocampo F, Camacho V, Fernández A, Ruiz A, et al. Comparison of image quality and lesion detection between digital and analog PET/CT. Eur J Nucl Med Mol Imaging 2019;46:1383-90.

12. van Sluis J, Boellaard R, Somasundaram A, van Snick P, Borra R, Dierckx $\mathrm{R}$, et al. Image quality and semi-quantitative measurements of the siemens biograph vision PET/CT: Initial experiences and comparison with siemens biograph mCT PET/CT. J Nucl Med 2020;61:129-35.

13. Nguyen NC, Vercher-Conejero JL, Sattar A, Miller MA, Maniawski PJ, Jordan DW, et al. Image quality and diagnostic performance of a digital PET prototype in patients with oncologic diseases: Initial experience and comparison with analog PET. J Nucl Med 2015;56:1378-85.

14. Senthamizhchelvan S, Bravo PE, Esaias C, Lodge MA, Merrill J, Hobbs RF, et al. Human biodistribution and radiation dosimetry of 82Rb. J Nucl Med 2010;51:1592-9.

15. Senthamizhchelvan S, Bravo PE, Lodge MA, Merrill J, Bengel FM, Sgouros G. Radiation dosimetry of $82 \mathrm{Rb}$ in humans under pharmacologic stress. J Nucl Med 2011;52:485-91.

16. Deak PD, Smal Y, Kalender WA. Multisection CT protocols: Sexand age-specific conversion factors used to determine effective dose from dose-length product. Radiol 2010;257:158-66.

17. van Dijk JD, Jager PL, Ottervanger JP, Slump CH, van Dalen JA. No need for frame-wise attenuation correction in dynamic rubidium-82 PET for myocardial blood flow quantification. J Nucl Cardiol 2019;26:738-45

18. Lortie M, Beanlands R, Yoshinaga K, Klein R, DaSilva J, deKemp R. Quantification of myocardial blood flow with $82 \mathrm{Rb}$ dynamic PET imaging. Eur J Nucl Med Mol Imaging 2007;34:1765-74.

19. Murthy V, Di Carli M. Non-invasive quantification of coronary vascular dysfunction for diagnosis and management of coronary artery disease. J Nucl Cardiol 2012;19:1060-72.
20. Koenders SS, van Dijk JD, Jager PL, Ottervanger JP, Slump CH, van Dalen JA. Impact of regadenoson-induced myocardial creep on dynamic rubidium-82 PET myocardial blood flow quantification. J Nucl Cardiol 2019;26:719-28.

21. Kitkungvan D, Johnson NP, Roby AE, Patel MB, Kirkeeide R, Gould KL. Routine clinical quantitative rest stress myocardial perfusion for managing coronary artery disease: Clinical relevance of test-retest variability. JACC Cardiovasc Imaging 2017;10:56577.

22. van Dijk JD, Jager PL, van Dalen JA. Pitfalls in myocardial blood flow quantification with rubidium-82 PET. Tijdschr voor Nucl Geneeskd 2017;39:1822-9.

23. Loghin C, Sdringola S, Gould KL. Common artifacts in PET myocardial perfusion images due to attenuation-emission misregistration: Clinical significance, causes, and solutions. J Nucl Med 2004;45:1029.

24. Martinez-Moller A, Souvatzoglou M, Navab N, Schwaiger M, Nekolla SG. Artifacts from misaligned CT in cardiac perfusion PET/CT studies: Frequency, effects, and potential solutions. J Nucl Med 2007;48:188.

25. Gould KL, Pan T, Loghin C, Johnson NP, Guha A, Sdringola S. Frequent diagnostic errors in cardiac PET/CT due to misregistration of CT attenuation and emission PET images: A definitive analysis of causes, consequences, and corrections. J Nucl Med 2007;48:1112-21

26. van Dijk J, Jager P, van Osch J, Khodaverdi M, van Dalen J. Comparison of maximal rubidium-82 activities for myocardial blood flow quantification between digital and conventional PET systems. J Nucl Cardiol 2019;26:1286-91.

27. Jødal L, Le Loirec C, Champion C. Positron range in PET imaging: An alternative approach for assessing and correcting the blurring. Phys Med Biol 2012;57:3931-43.

28. Renaud JM, Yip K, Guimond J, Trottier M, Pibarot P, Turcotte E, et al. Characterization of 3-dimensional PET systems for accurate quantification of myocardial blood flow. J Nucl Med 2017;58:1039.

29. deKemp RA, Yoshinaga K, Beanlands RSB. Will 3-dimensional PET-CT enable the routine quantification of myocardial blood flow? J Nucl Cardiol 2007;14:380-97.

30. Tahari AK, Lee A, Rajaram M, Fukushima K, Lodge MA, Lee $\mathrm{BC}$, et al. Absolute myocardial flow quantification with (82)rb PET/CT: Comparison of different software packages and methods. Eur J Nucl Med Mol Imaging 2014;41:126-35.

31. Armstrong I, Tonge C, Arumugam P. Impact of point spread function modeling and time-of-flight on myocardial blood flow and myocardial flow reserve measurements for rubidium-82 cardiac PET. J Nucl Cardiol 2014;21:467-74.

Publisher's Note Springer Nature remains neutral with regard to jurisdictional claims in published maps and institutional affiliations. 